\title{
VALORACIÓN DEL FUNCIONAMIENTO COGNITIVO EN MUJERES CON CÁNCER DE MAMA ANTES DE INICIAR EL TRATAMIENTO ONCOLÓGICO
}

\author{
ASSESSMENT OF COGNITIVE FUNCTIONING IN WOMEN WITH BREAST CANCER \\ BEFORE STARTING TREATMENT CANCER
}

\begin{abstract}
Beatriz Rubio', Agustina Sirgo', Sergio Castillo², Josep Creus², Dolores Martín ${ }^{2}$ y Josep Gumà2

' Unidad de Psico-oncología. Área de Oncología. Hospital Universitari Sant Joan de Reus (Tarragona)

2 Área de Oncología. Hospital Universitari Sant Joan de Reus (Tarragona)
\end{abstract}

Resumen

Objetivo: El principal objetivo es describir el rendimiento cognitivo previo al tratamiento oncológico sistémico en mujeres con cáncer de mama dentro de un estudio longitudinal.

Método: A 35 mujeres diagnosticadas de cáncer de mama no metastásico, con una edad media de 48,6 años, se les ha realizado una evaluación neurocognitiva, sobre diferentes dominios cognitivos, y psicológica (malestar emocional y percepción subjetiva de fallos cognitivos) antes de iniciar el tratamiento.

Resultados: El 22,9\% de la muestra precisa quimioterapia, el $28,6 \%$ hormonoterapia y el $48,6 \%$ un tratamiento combinado. Los resultados muestran que: (1) Entre un $62 \%$ y un $100 \%$ presenta un rendimiento normal en los distintos dominios cognitivos, excepto en memoria visual donde sólo un $43 \%$ cumple criterios de normalidad. (2) Aparece deterioro cognitivo leve en atención selectiva $(23,5 \%)$ y memoria visual $(14,4 \%)$, y deterioro cognitivo severo en atención y velocidad de procesamiento $(28,6 \%)$ y memoria visual $(22,9 \%)$. (3) Un porcentaje reducido de mujeres presenta niveles elevados de depresión y ansiedad (2,9\% y $14,5 \%$, respectivamente). (4) El $71 \%$ manifiesta baja percepción subjetiva de fallos cognitivos. (5) Los tests neurocognitivos y las variables psicológicas son independientes. (6)

\section{Abstract}

Objective: The aim of this study is to describe the cognitive achievement previous to oncological treatment in a group of breast cancer women included in a longitudinal study.

Method: Thirty five non metastatic breast cancer women, with a mean age of $\mathbf{4 8 . 6}$ years old, were assessed in different neurocognitive domains and also in some psychological variables (emotional distress and subjective perception of cognitives failures) before to start the oncological treatment.

Results: Twenty two percent of the group received chemotherapy alone, $28.6 \%$ hormonotherapy and $48.6 \%$ a mixed treatment (chemotherapy plus hormonotherapy). Results show that: (1) Bewteen a $62 \%$ and a $100 \%$ of the women present a normal achievement in the different cognitive domains, except for visual memory where only $43 \%$ gets into the normality. (2) There is a light cognitive impairment in selective attention $(23.5 \%)$ and visual memory $(14.4 \%)$, also there is a severe cognitive impairment in attention and processing speed $(28.6 \%)$ and visual memory $(22.9 \%)$. (3) A low percentage of women shows high levels of emotional distress, $2.9 \%$ for depression and $\mathbf{1 4 . 5} \%$ for anxiety. (4) The $71 \%$ reports a low subjective perception of cognitives failures. (5) Neurocogntive test and psychological variables

\section{Correspondencia:}


La percepción subjetiva del funcionamiento cognitivo correlaciona con la ansiedad ( $r=$ $-0,409$ ).

Conclusión: Un número reducido de pacientes presenta deterioro cognitivo, antes de iniciar el tratamiento, de estos un porcentaje muestra deterioro severo. Es indispensable contar con una línea base de funcionamiento cognitivo para evaluar correctamente a estos pacientes y habría que revisar la metodología usada y la idoneidad de las pruebas neurocognitivas administradas.

Palabras Clave: Cáncer de mama, quimioterapia, deterioro cognitivo, evaluación neurocognitiva. do not show any correlation. (6) Subjective perception of cognitive failure correlates with anxiety $(r=-0.409)$.

Conclusion: $A$ reduced number of patients shows cognitive impairment before to start the oncological treatment, a small percentage of this group shows severe impairment. It is very important to have a baseline of cognitive functioning to correctly assess these patients. It is also necesary to review the methodology and the neurocognitive test used.

Key Words: Breast cancer, chemotherapy, cognitive impairment, neurocognitive assessment.

\section{INTRODUCCIÓN}

Desde los años 90 se ha comenzado a prestar una mayor atención a la calidad de vida de los pacientes con cáncer, lo que ha favorecido que surgieran los primeros estudios referentes al efecto que los tratamientos oncológicos sistémicos, especialmente la quimioterapia, tienen sobre el funcionamiento cognitivo del paciente ${ }^{(1-2)}$. Estos estudios eran muy rudimentarios en su diseño y su tamaño muestral carecía del suficiente poder estadístico para extraer conclusiones, pero sirvieron para tomar conciencia del tema y despertar el interés de los investigadores.

La mayor investigación se ha realizado en mujeres con cáncer de mama. Estas pacientes son las que verbalizan más abiertamente en consulta sus quejas y es una de las poblaciones oncológicas en la que se obtienen cifras de supervivencia más elevadas. Así, desde que surgieron los primeros estudios sobre el tema ${ }^{(1,2)}$ ha habido un crecimiento exponencial de las publicaciones, lo que indica un interés creciente por el tema en la actualidad.

A lo largo de los años ha habido una evolución en el diseño de estas investigaciones. Los primeros estudios ${ }^{(2-8)}$ eran de carácter transversal y la evaluación del funcionamiento cognitivo se realizaba en un único momento temporal, bien sea durante o tras la administración de la quimioterapia. Este momento era muy variable entre estudios, lo que dificultaba la comparación de los resultados.

A partir del año 2000 se empiezan a publicar los primeros estudios longitudinales y prospectivos ${ }^{(9-11)}$ y disminuye de forma progresiva el número de estudios transversales. A su vez se destaca la importancia de introducir una primera evaluación pretratamiento que sirva de línea base con la que comparar posteriores resultados. Esta primera evaluación permite estudiar con exactitud la verdadera magnitud del cambio observado tras la administración de un tratamiento oncológico sistémico, permitiendo a su vez estudiar su evolución a través del tiempo (estabilidad, mejoría o empeoramiento). Su ausencia puede conllevar a una sobrevaloración o infravaloración del deterioro cognitivo.

La importancia de realizar esta primera evaluación queda de manifiesto en el estudio de Wefel et al. ${ }^{(9)}$, un estudio longitudinal en el que se incluye una evaluación pretratamiento y que concluye que el deterioro cognitivo puede estar presente antes del inicio de los tratamientos. Sus resultados indican que el 35\% de la mues- 
tra presenta deterioro cognitivo, siendo el aprendizaje verbal (18\%) y la memoria (25\%) los dominios más afectados.

Otros estudios que también muestran la presencia de deterioro cognitivo en la línea base son el estudio de Hurria et al. ${ }^{(12)}$ y de Hermelink et al. ${ }^{(13)}$. En el primero(12) un $11 \%$ de la muestra presentaba deterioro cognitivo en la línea base y en el segun$\mathrm{do}^{(13)}$ la media del grupo tenía un rendimiento inferior a los datos normativos en 5 de los 12 tests administrados para evaluar el funcionamiento cognitivo.

Tras revisar la literatura disponible sobre este tópico y dadas las quejas realizadas por algunas pacientes en la práctica clínica hospitalaria, surgió el interés de desarrollar un estudio, tratando de superar algunas de las limitaciones metodológicas observadas en estudios precedentes.

\section{OBJETIVOS E HIPÓTESIS}

El objetivo principal de este trabajo es exponer los resultados referentes al funcionamiento cognitivo de un grupo de mujeres con cáncer de mama no metastásico previo al inicio de un tratamiento oncológico sistémico (quimioterapia, hormonoterapia o ambos). Esta primera evaluación actuaría como la línea base del funcionamiento cognitivo de esta muestra, siendo fundamental para establecer comparaciones con los resultados obtenidos en las evaluaciones neuropsicológicas realizadas tras finalizar los tratamientos. La ausencia de esta línea base puede conllevar a una infravaloración o sobrestimación del deterioro inducido por la quimioterapia y/o tratamiento hormonal, ya que el deterioro cognitivo puede estar presente antes de la recepción de los tratamientos ${ }^{(9,12,13)}$.

Nuestra hipótesis de partida es que en esta primera evaluación pretratamiento (línea base) un porcentaje elevado de mujeres va tener un rendimiento cognitivo dentro de la normalidad y que el porcenta- je de mujeres con deterioro cognitivo va a ser muy reducido. A su vez, se espera que el deterioro cognitivo observado sea leve (no severo e incapacitante) y específico de algunos dominios cognitivos.

Como hipótesis secundaria, no esperamos encontrar correlación entre el funcionamiento cognitivo objetivo, es decir, evaluado mediante tests neuropsicológicos, y la percepción subjetiva que la persona tiene sobre su rendimiento cognitivo, considerando estas variables independientes.

\section{MÉTODO}

\section{Diseño del estudio y recogida de datos}

Aunque en el presente trabajo sólo se presentan los resultados referentes a la evaluación pretratamiento, éstos forman parte de un estudio longitudinal y prospectivo, todavía en proyecto, en el que se evalúa el funcionamiento cognitivo en tres momentos temporales distintos [una primera evaluación pre-tratamiento y dos evaluaciones postratamiento (a 6 y 18 meses)].

Las mujeres son invitadas a participar tras su primer contacto con el oncólogo. En este primer contacto es cuando se determina el tratamiento oncológico requerido por la paciente. Se invita a participar sólo a aquellas mujeres diagnosticadas de cáncer de mama en un estadio no metastásico que precisan un tratamiento sistémico adyuvante o neoadyuvante.

La invitación a participar se realiza telefónicamente. El psico-oncólogo, tras presentarse, explica la finalidad del estudio y solicita la colaboración voluntaria de la paciente. Si accede, se establece un día para la evaluación. Este día, antes de realizar la evaluación, se explica nuevamente la finalidad del estudio y sus características y se firma una hoja de consentimiento informado.

En el caso de las mujeres que precisan quimioterapia, el planteamiento inicial era 
realizar la evaluación del funcionamiento neurocognitivo antes de recibir el primer ciclo de quimioterapia pero por diversas cuestiones prácticas (dificultad para compaginar actividad asistencial y de investigación, reducido intervalo de tiempo entre visita oncólogo e inicio de los tratamientos, múltiples pruebas médicas los días previos al tratamiento) muchas de las mujeres candidatas tenían que ser excluidas del estudio al haber iniciado ya el tratamiento. Ante esta situación, decidimos incluir a las mujeres aún cuando ya se hubiesen sometido a un primer ciclo de quimioterapia, pensando que un sólo ciclo no tendría repercusión sobre el funcionamiento cognitivo.

En el caso de las mujeres sometidas a tratamiento hormonal la evaluación se debía realizar dentro del primer mes desde el inicio del tratamiento. En este grupo de mujeres no hubo problemas para ajustarse a este criterio.

La evaluación incluye una serie de pruebas neuropsicológicas y psicológicas, que se administran de forma individualizada. El tiempo requerido para la administración de estas pruebas oscila entre 120 y 150 minutos. Estas pruebas, así como su orden de administración, se detallan en posteriores apartados.

Además de la administración de estas pruebas, se recogen datos sociodemográficos, antecedentes médicos y su historia oncológica.

Las variables sociodemográficas evaluadas son: edad, estado civil, número de hijos, nivel académico, profesión, situación laboral.

Dentro de antecedentes médicos se incluye: el estado de menopausia en el momento de la evaluación, la presencia de antecedentes psiquiátricos, así como la presencia de otras enfermedades concomitantes al cáncer y sus respectivos tratamientos médicos, la historia de accidente cerebral, epilepsia y/o dificultades de aprendizaje en la infancia.
En cuanto a la historia oncológica se recoge: subtipo de cáncer de mama, el estadio, tratamiento sistémico adyuvante recibido (quimioterapia, tratamiento hormonal o tratamiento combinado), el esquema de quimioterapia recibido, junto con su dosis y número de ciclos, y el tipo de tratamiento hormonal requerido.

Una vez administradas las pruebas y recogidos todos los datos, éstos son informatizados mediante la base de datos SPSS (versión 15.0).

\section{Población de estudio y tamaño muestral}

El grupo de pacientes se compone de mujeres de entre 26 y 65 años recientemente diagnosticadas de cáncer de mama en un estadio no metastásico que además de los tratamientos locales (cirugía y radioterapia a nivel de la mama) precisan un tratamiento adyuvante sistémico con quimioterapia, con tratamiento hormonal o una combinación de los anteriores.

Debido a este amplio rango de edad, la muestra estará compuesta por mujeres que en el momento del diagnóstico son premenopáusicas, perimenopáusicas, o postmenopáusicas, estableciendo subgrupos en función de su estado hormonal.

Del mismo modo, se podrán establecer subgrupos en función de la modalidad de tratamiento requerido (tratamiento exclusivo con quimioterapia, tratamiento hormonal o tratamiento sistémico combinado).

\section{Criterios de exclusión}

Como criterios de exclusión se han establecido los siguientes:

- Una edad inferior a 18 años o superior a 65 años.

- Haber iniciado la quimioterapia o estar tomando el tratamiento hormonal desde más de un mes.

- Precisar únicamente tratamiento local con radioterapia y/o cirugía. 
- Historia de traumatismo cerebral, epilepsia u otras enfermedades.

- Historia de dificultades de aprendizaje.

- Historia de abuso de alcohol u otras drogas.

- Historia de trastornos psiquiátricos graves.

- Demencia.

- No ser castellano parlante.

- Haber padecido una enfermedad neoplásica en el pasado, precisando quimioterapia.

- Padecer un cáncer de mama en un estadio avanzado o en otras localizaciones distintas a la mama.

\section{Instrumentos de evaluación}

A continuación se describen los instrumentos neurocognitivos utilizados en el estudio para evaluar las diferentes funciones cognitivas objeto de interés, así como los instrumentos de autoinforme administrados para la valoración de la calidad de vida, nivel de fatiga, presencia de estados de ansiedad y/o depresión y percepción subjetiva del funcionamiento cognitivo.

A la hora de seleccionar los instrumentos de evaluación neuropsicológica nos hemos guiado por los instrumentos utilizados en estudios previos sobre el tema, seleccionando aquellos con mejores propiedades psicométricas, es decir, que cuenten con una buena fiabilidad, validez, sensibilidad y especificidad, y que a su vez dispongan de una versión adaptada y validada al castellano y sean de fácil administración.

De este modo, todos los instrumentos utilizados, a excepción del Test Complutense de Aprendizaje Verbal (TAVEC) ${ }^{(14)}$ y el Test breve de Inteligencia (K-BIT) $)^{(15)}$, han sido utilizados en estudios precedentes. El TAVEC ${ }^{(14)}$ es un instrumento para la evaluación del aprendizaje y memoria verbal originariamente desarrollado en castellano, siendo muy similar en su diseño al California Verbal Learning Test $(C V L T)^{(16)}$, test del que no disponemos versión en castellano. El K-BIT ${ }^{(15)}$ es un test adaptado al español por Cordero y Calonge (2000), con buenas propiedades psicométricas y que ha sido seleccionado por su brevedad con respecto a otros tests destinados a la evaluación de la inteligencia, como por ejemplo La Escala de Inteligencia para adultos de Weschler $-3^{\text {a }}$ versión ${ }^{(17)}$.

Las funciones cognitivas evaluadas en nuestro estudio son: la inteligencia, la capacidad de atención/concentración, la memoria verbal y visual, el lenguaje (concretamente la fluencia verbal), la velocidad de procesamiento de la información, la función ejecutiva y la capacidad visuoconstructiva. Nos hemos centrado en estas funciones cognitivas por ser las que con más frecuencia se muestran afectadas en los diversos estudios, a pesar de las múltiples inconsistencias entre los mismos.

Estas funciones o dominios cognitivos han sido evaluados mediante las siguientes pruebas (ver Tabla 1).

Respecto a las pruebas psicológicas se han seleccionado aquellas que evalúan variables que pueden modular el rendimiento de las pruebas neurocognitivas, como son la fatiga y la presencia de sintomatología ansiosa y depresiva.

A su vez también se ha introducido una medida de calidad de vida y de la percepción subjetiva de deterioro cognitivo. Esta última variable ha sido incluida con el fin de establecer la asociación entre la percepción subjetiva y el rendimiento objetivo, dado que numerosos estudios muestran que no hay correlación entre ambos, lo que destaca la necesidad de que el rendimiento cognitivo sea evaluado a través de pruebas neuropsicológicas.

Todas las pruebas de autoinforme seleccionadas para el estudio disponen de una versión adaptada y validada en castellano. A excepción de la Escala de Depresión 
Tabla 1. Tests Neurocognitivos utilizados

\begin{tabular}{|c|c|}
\hline \multicolumn{2}{|r|}{ FUNCIONES COGNITIVAS } \\
\hline $\begin{array}{l}\text { Atención selectiva/resistencia a } \\
\text { la monotonía: }\end{array}$ & $\begin{array}{l}\text { - Subtest de Dígitos del WAIS- III (Weschler, 2001 }{ }^{(17)} \text {. } \\
\text { - TMT- A (Reitan y Wolsfon, 1993) } \\
\\
\text { - Test de Atención de Tolouse-Pierón (1994) }\end{array}$ \\
\hline $\begin{array}{l}\text { MEMORIA } \\
\text { - Memoria de trabajo: } \\
\text { - Memoria visual: } \\
\text { - Memoria verbal/aprendizaje: }\end{array}$ & $\begin{array}{l}\text { - Subtest de números y letras del WAIS- III (Weschler, 2001) }{ }^{(17)} \text {. } \\
\text { - Subtest de Dígitos del WAIS- III (Weschler, 2001 } 1^{(17)} \text {. } \\
\text { - TMT-B (Reitan y Wolsfon, 1993) } \\
\text { - Figura Compleja de Rey - Recuerdo (Meyers y Meyers, 1995) } \\
\text { - TAVEC de Benedet y Alejandre }(1998)^{(14)} \text {. }\end{array}$ \\
\hline $\begin{array}{l}\text { FUNCIÓN EJECUTIVA: } \\
\text { - Razonamiento abstracto: } \\
\text { - Planificación: } \\
\text { - Secuenciación: }\end{array}$ & $\begin{array}{l}\text { - Subtest de Semejanzas del WAIS-III (Weschler, 2001)(17). } \\
\text { - Subtest de Matrices del K-BIT de Kauffman }(1994)^{(15)} \text {. } \\
\text { - Figura Compleja de Rey - Copia (Meyers y Meyers, 1995) } \\
\text { - TMT-B (Reitan y Wolsfon, 1993) }\end{array}$ \\
\hline $\begin{array}{l}\text { LENGUAJE: } \\
\text { - Fluencia verbal: }\end{array}$ & 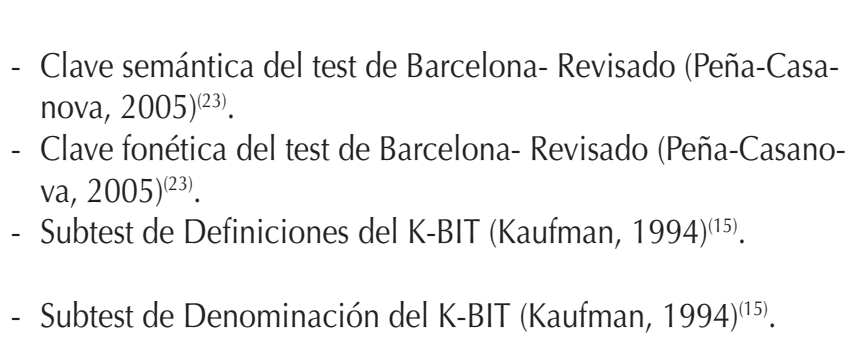 \\
\hline $\begin{array}{l}\text { VELOCIDAD DE } \\
\text { PROCESAMIENTO: }\end{array}$ & $\begin{array}{l}\text { - Clave de números del WAIS - III (Weschler, 2001) }{ }^{(17)} \text {. } \\
\text { - TMT-A (Reitan y Wolsfon, 1993) } \\
\text { - Figura Compleja de Rey - Tiempo de copia (Meyers y Me- } \\
\text { yers, } 1995)^{(20)} \text {. }\end{array}$ \\
\hline INTELIGENCIA & - K-BIT (Kaufman, (1994) $)^{(15)}$. \\
\hline CAPAC. VISOCONSTRUCCIÓN & - Figura Compleja de Rey - Copia (Meyers y Meyers, 1995) \\
\hline
\end{tabular}

WAIS-III: Escala de Inteligencia para adultos (3ª edición) de Weschler (2001); TMT-A: Trail Making Test- Parte A de Reitan y Wolsfon (1993); TMT-B: Trail Making Test- Parte B de Reitan y Wolsfon (1993); TAVEC: Test Complutense de Aprendizaje Verbal de Benedet y Alejandre (1998); K-BIT: Escala breve para la evaluación de la inteligencia de Kauffman (1994). 
Tabla 2. Cuestionarios psicológicos utilizados

\begin{tabular}{|l|l|}
\hline \multicolumn{2}{|c|}{ VARIABLES PSICOLÓGICAS } \\
\hline ANSIEDAD: & - Subescala de Estado del STAI de Spielberg et al. (1986). \\
\hline DEPRESIÓN: & - Escala para la depresión de Zung (Conde et al., 1970). \\
\hline CALIDAD DE VIDA: & - SF-36 (Alonso et al., 2003). \\
\hline FATIGA: & $\begin{array}{l}\text { - Escala de fatiga/ cansancio del FACIT (4ª Versión) de Cella } \\
\text { et al. (1993). }\end{array}$ \\
\hline $\begin{array}{l}\text { PERCEPCIÓN SUBJETI- } \\
\text { VA DE DÉFICITS COG- } \\
\text { NITIVOS: }\end{array}$ & - CFQ de Broadbent et al. (1982). \\
\hline
\end{tabular}

STAI: Inventario de Ansiedad Estado - Rasgo; SF-36: Cuestionario de Salud; FACIT: Functional Assessment of Cancer Therapy; CFQ: Cuestionario de Fallos Cognitivos.

de Zung ${ }^{(18)}$ y El Cuestionario de Salud SF$36^{(19)}$, las otras pruebas han sido utilizadas en los estudios precedentes sobre el tema.

En la Tabla 2 se recogen los cuestionarios psicológicos utilizados.

Estas pruebas (neurocognitivas y psicológicas) son administradas en un orden predeterminado. Este orden es el siguiente:

1. Test Complutense de Aprendizaje Verbal (TAVEC): Aprendizaje y recuerdo a corto plazo libre y con claves ${ }^{(14)}$

2. Figura Compleja de Rey: copia ${ }^{(20)}$.

3. Prueba perceptiva y de atención (21).

4. Test del trazo: Parte $\mathrm{A}^{(22)}$.

5. Test del trazo: Parte $\mathrm{B}^{(22)}$.

6. TAVEC: recuerdo a largo plazo libre y con claves y reconocimiento ${ }^{(14)}$.

7. Figura Compleja de Rey: recuerdo ${ }^{(20)}$.

8. Test Breve de Inteligencia de Kaufman $(\mathrm{K}-\mathrm{BIT})^{(15)}$.

9. Subtest de Dígitos (directos e indirectos) de la Escala de Inteligencia para adultos de Weschler- $3^{\text {a }}$ versión (WAIS- III) $)^{(17)}$.

10. Subtest de Clave Fonética del Test de Barcelona - Revisado. Programa Integrado de Exploración Neuropsicológica ${ }^{(23)}$

11. Subtest de Clave Semántica del Test de Barcelona- Revisado ${ }^{(23)}$.

12. Subtest de Semejanzas del WAISIII (17).
13. Subtest de Letras y Números del WAIS-III ${ }^{(17)}$.

14. Subtest de Clave de Números del WAIS-III (17).

15. Escala para la Depresión de Zung ${ }^{(18)}$.

16. Subescala de Estado de Ansiedad del Inventario de Ansiedad Estado-Ras$\mathrm{go}^{(24)}$.

17. Escala de Fatiga del Functional Assessment of Cancer Therapy (FACIT) $\left(4^{\mathrm{a}}\right.$ versión) $)^{(25)}$.

18. Cuestionario de Salud SF-36. Versión adaptada y validada al español del Health Survey SF-36 ${ }^{(19)}$.

19. Cuestionario de Fallos Cognitivos ${ }^{(26)}$.

\section{Análisis de datos}

Los datos recogidos a través de los cuestionarios se informatizan para poder ser analizados mediante el programa estadístico SPSS (15.0).

Los resultados obtenidos en los tests neurocognitivos se comparan con los datos normativos $y$, atendiendo a los criterios establecidos de deterioro cognitivo, se establece su presencia, así como el grado de deterioro.

También se realiza un estudio de correlaciones entre los diferentes dominios 
cognitivos evaluados, las variables psicológicas consideradas y algunas de las variables sociodemográficas recogidas, como es el nivel de estudios y la edad, tratando de ver la relación existente entre las diversas variables. Concretamente, mediante el análisis de correlaciones queremos someter a prueba nuestra segunda hipótesis, referente a la ausencia de relación entre las medidas objetivas del funcionamiento cognitivo y las medidas subjetivas (percepción de fallos cognitivos).

Los criterios utilizados para establecer la presencia de deterioro cognitivo en este trabajo han sido los siguientes ${ }^{(27)}$ :

A.- Ausencia de deterioro cognitivo.

Diremos que no hay deterioro cognitivo en un dominio cognitivo cuando la puntuación obtenida por la persona se encuentra dentro de la normalidad, es decir, \pm 1.5 desviación estándar (DE) respecto de la media. leve.

B.- Presencia de deterioro cognitivo

Respecto a la presencia de deterioro cognitivo leve, la mayoría de los investigadores de forma implícita (puesto que no está basado en una reunión de consenso internacional) aceptan que cuando el rendimiento en un dominio está por debajo de 1.5DE, el dominio está alterado y afectado de forma leve. En cambio otros autores utilizan criterios mucho más sensibles y fijan el límite en 1DE.

Otro aspecto a tener en cuenta a la hora de establecer la presencia de deterioro en un dominio cognitivo específico es, no sólo la magnitud del rendimiento deficitario, sino también el número de pruebas neuropsicológicas que evalúan un determinado dominio que deben mostrar un rendimiento inferior a 1,5DE ó $1 \mathrm{DE}$ respecto a la media para considerarlo afectado.

Respecto a esta última cuestión tampoco hay un acuerdo entre investigadores, ya que depende de muchos factores, como son la sensibilidad y especificidad de los diferentes tests utilizados, sus características psicométricas, la disponibilidad de unos valores normativos representativos de la población de referencia y adaptados a la población española.

A pesar de ello, generalmente la definición de un dominio afectado se basa en la obtención de un resultado en una prueba cognitiva que valore adecuadamente ese dominio, por debajo de 1,5DE (ó 1DE) para sus valores normativos por edad y escolaridad.

Acogiéndonos a estas definiciones, en este estudio el criterio para establecer la presencia de deterioro cognitivo leve en un dominio cognitivo específico implicará obtener una puntuación por debajo de 1.5DE en al menos un test destinado a evaluar dicho dominio.

\section{C.- Presencia de deterioro cognitivo se- vero.}

Respecto a la presencia de deterioro cognitivo severo, la mayoría de autores consideran que la puntuación del sujeto debe situarse por debajo de 2DE respecto de la media.

De este modo, consideraremos que una persona presenta deterioro cognitivo severo en un dominio cognitivo si su rendimiento es inferior a 2DE respecto a la media de los datos normativos.

\section{Resultados preliminares del estudio}

En las tablas 3 y 4 se recogen las características sociodemográficas y médicas consideradas relevantes para el estudio de las 35 pacientes evaluadas.

El $71,5 \%$ de la muestra realiza quimioterapia. De este $71,5 \%$, sólo un $17,14 \%$ $(n=6)$ han sido evaluadas antes de realizar el primer ciclo. El resto del grupo (54,3\%) ha sido evaluado tras la aplicación del primer ciclo de quimioterapia. 
Tabla 3. Características sociodemográficas $(\mathbf{N}=\mathbf{3 5}$ )

\begin{tabular}{|l|c|}
\hline EDAD (años) & \\
Media (DE): & $48,57(10,1)$ \\
Rango: & $26-65$ \\
\hline NIVEL EDUCATIVO (\%) \\
Primarios: & 48,6 \\
Secundarios: & 28,6 \\
Universitarios: & 22,8 \\
\hline ESTADO DE MENOPAUSIA (\%) & 62,9 \\
Premenopausia: & 0 \\
Perimenopausia: & 37,1 \\
Postmenopausia: & \\
\hline
\end{tabular}

Tabla 4. Características médicas ( $\mathbf{N}=\mathbf{3 5}$ )

\begin{tabular}{|c|c|}
\hline Tratamiento oncológico sistémico (\%): & \\
- Quimioterapia Neoadyuvante. & 14,3 \\
- Quimioterapia Adyuvante. & 8,6 \\
- Tratamiento Hormonal. & 28,6 \\
- Tratamiento combinado. & 48,6 \\
\hline Quimioterapia (\%): & 71,5 \\
- Evaluación pre-quimioterapia: & 17,14 \\
- Evaluación tras 1 ${ }^{\text {er }}$ ciclo: & 54,3 \\
\hline Cirugía (\%): & \\
- Tumorectomia. & 51,4 \\
- Mastectomia. & 25,7 \\
- Pendiente de cirugía. & 22,9 \\
\hline
\end{tabular}

\section{Tabla 5. Variables psicológicas $(\mathbf{N}=\mathbf{3 5}$ )}

\begin{tabular}{|c|c|}
\hline DEPRESIÓN: & Escala para la Depresión de Zung (\%): \\
- $\quad$ Ausencia de depresión. & 23,5 \\
$-\quad$ Depresión leve. & 51,6 \\
- Depresión moderada. & 22 \\
$-\quad$ Depresión severa. & 2,9 \\
\hline ANSIEDAD: & Subescala de Estado de Ansiedad del STAI (\%): \\
- Ansiedad elevada. & 14,5 \\
- Ansiedad normal. & 73,9 \\
- Ansiedad baja. & 11,6 \\
\hline PERCEPCIÓN DE FALLOS COGNI- & Cuestionario de Fallos Cognitivos de \\
TIVOS: & Broadbent (\%): \\
- Elevada. & 5,8 \\
- Moderada. & 22,8 \\
- Baja. & 71,4 \\
\hline
\end{tabular}


Tabla 6. Porcentajes de deterioro para cada test neuropsicológico utilizado

\begin{tabular}{|c|c|c|c|}
\hline DOMINIO COGNITIVO & $\begin{array}{l}\text { Normalidad } \\
(\%)\end{array}$ & $\begin{array}{c}\text { Deterioro } \\
\text { cognitivo leve } \\
(\%)\end{array}$ & $\begin{array}{c}\text { Deterioro } \\
\text { cognitivo severo } \\
(\%)\end{array}$ \\
\hline $\begin{array}{l}\text { INTELIGENCIA GENERAL: } \\
\text { - K-BIT - Total. }\end{array}$ & 77,1 & 2,9 & 0 \\
\hline $\begin{array}{l}\text { ATENCIÓN: } \\
\text { - General: } \\
\text { - Dígitos (WAIS-III). } \\
\text { - TMT-A. } \\
\text { - Selectiva: } \\
\text { - Test de Tolouse-Piéron. }\end{array}$ & $\begin{array}{l}74,3 \\
68,5 \\
62,9\end{array}$ & $\begin{array}{r}2,9 \\
2,9 \\
23,5\end{array}$ & $\begin{array}{c}0 \\
28,6 \\
8,8\end{array}$ \\
\hline $\begin{array}{l}\text { MEMORIA: } \\
\text { - Memoria de trabajo: } \\
\text { - Letras y números (WAIS-III). } \\
\text { - Dígitos (WAIS-III). } \\
\text { - TMT-B. } \\
\text { - Memoria visual: } \\
\text { - Recuerdo del ROCFT. } \\
\text { - Memoria verbal: } \\
\text { - TAVEC: } \\
\text { Aprendizaje. } \\
\text { Recuerdo libre (corto plazo). } \\
\text { Recuerdo con claves (corto plazo). } \\
\text { Recuerdo libre (largo plazo). } \\
\text { Recuerdo con claves (largo plazo). } \\
\text { Reconocimiento. }\end{array}$ & $\begin{array}{l}88,6 \\
97,1 \\
88,6 \\
94,2 \\
94,3 \\
85,7\end{array}$ & $\begin{array}{c}0 \\
0 \\
2,9 \\
2,9 \\
2,9 \\
5,7\end{array}$ & $\begin{array}{c}0 \\
0 \\
11,4 \\
22,9\end{array}$ \\
\hline $\begin{array}{l}\text { LENGUAJE: } \\
\text { - Fluencia verbal y denominación: } \\
\text { - Clave Fonética (Test de Barcelona). } \\
\text { - Clave Semántica (Test Barcelona). } \\
\text { - Vocabulario (K-BIT). }\end{array}$ & $\begin{array}{l}82,8 \\
80 \\
68,5\end{array}$ & $\begin{array}{c}2,9 \\
5,7 \\
0\end{array}$ & $\begin{array}{l}5,7 \\
11,4 \\
0\end{array}$ \\
\hline $\begin{array}{l}\text { VISUOCONSTRUCCIÓN: } \\
\text { - Copia del ROCFT. }\end{array}$ & 68,6 & 5,7 & 5,7 \\
\hline $\begin{array}{l}\text { VELOCIDAD DE PROCESAMIENTO: } \\
\text { - Clave de Números (WAIS-III). } \\
\text { - TMT-A. } \\
\text { - Tiempo de Copia (ROCFT). }\end{array}$ & $\begin{array}{l}84,7 \\
68,5 \\
94,2\end{array}$ & $\begin{array}{l}2,9 \\
2,9 \\
2,9\end{array}$ & $\begin{array}{c}2,9 \\
28,6 \\
2,9\end{array}$ \\
\hline $\begin{array}{l}\text { FUNCIONES EJECUTIVAS: } \\
\text { - Pensamiento Abstracto: } \\
\text { - Semejanzas (WAIS-III). } \\
\text { - Matrices (K-BIT). } \\
\text { - Planificación. } \\
\text { - Tipo de copia (ROCFT). } \\
\text { - Clave semántica/serial } \\
\text { (TAVEC). } \\
\text { - Secuenciación. } \\
\text { - TMT-B. }\end{array}$ & $\begin{array}{c}74,3 \\
62,9 \\
68,6 \\
94,3 / 88,6\end{array}$ & $\begin{array}{c}0 \\
2,9 \\
5,7 \\
0\end{array}$ & $\begin{array}{c}0 \\
0 \\
5,7 \\
0 \\
11,4\end{array}$ \\
\hline
\end{tabular}


En la tabla 5 se muestran los datos referentes a las variables psicológicas evaluadas.

Como se puede observar en la tabla, un porcentaje elevado de mujeres muestra síntomas depresivos de carácter leve $(51,6 \%)$ o moderado $(22 \%)$, y sólo un reducido porcentaje presenta síntomas depresivos severos (2,9\%). Aproximadamente un cuarto de la muestra (23.5\%) no presenta sintomatología depresiva.

En relación a la ansiedad, se obtiene que el $74 \%$ de las pacientes muestra niveles de ansiedad normales y aproximadamente un 15\% muestra una ansiedad elevada.

Por último, las puntuaciones referentes a la percepción de fallos cognitivos muestran que el $71 \%$ de mujeres perciben que tienen un buen rendimiento cognitivo en su vida diaria, es decir, un reducido número de fallos cognitivos, mientras que sólo un $6 \%$ percibe que tiene fallos cognitivos frecuentes.

En lo referente a la evaluación neuropsicológica, en la tabla 6 se recogen los distintos dominios cognitivos evaluados, así como los tests utilizados para su evaluación, y se muestran mediante porcentajes el número de pacientes que tienen un rendimiento cognitivo considerado normal, así como los porcentajes de pacientes que presentan deterioro cognitivo leve o severo, según los criterios previamente establecidos en cada test.

También hay un reducido porcentaje de la muestra que presenta un rendimiento superior a la media en cada uno de los tests. Estos porcentajes no se recogen en la tabla debido a que no se consideran relevantes para el estudio.

Como se puede ver en la tabla 6, los resultados muestran que un porcentaje elevado de las mujeres tienen un rendimiento similar al de la población general en los diferentes dominios cognitivos. De hecho, se obtienen porcentajes superiores a $62 \%$, por ejemplo en atención y visuoconstrucción, y cercanos al 100\% en algunos dominios cognitivos como, por ejemplo, la memoria verbal. Sólo en el test empleado para la evaluación de la memoria visual, el porcentaje de pacientes que muestra un rendimiento dentro de la normalidad es reducido $(43 \%)$.

También se puede observar que a pesar del buen rendimiento mostrado por un número importante de mujeres, una proporción reducida de ellas, pero superior a la esperada, muestra también deterioro en algunos tests, cumpliendo criterios de deterioro cognitivo leve y severo.

En un buen número de tests, y en consecuencia en todos los dominios cognitivos, se observa la presencia de deterioro cognitivo leve, destacando los porcentajes obtenidos en atención selectiva $(23,5 \%)$ y memoria visual $(14,4 \%)$ frente a los restantes. En el resto de pruebas la presencia de deterioro cognitivo oscila entre porcentajes relativamente bajos (entre 0 y 5,8\%).

En cuanto a la presencia de deterioro cognitivo severo, vemos que no aparece en todos los tests ni dominios cognitivos, pero en aquellos en los que se presenta, alcanza porcentajes superiores a los de deterioro cognitivo leve. El deterioro cognitivo severo oscila entre 2,9\% y 28,6\%. Los tests en los que se observan mayores porcentajes de deterioro severo son el Trail Making Test- Parte $A^{(22)}(28,6 \%)$ para la evaluación de la atención y velocidad de procesamiento, el recuerdo en la Figura Compleja de $\operatorname{Rey}^{(20)}(22,9 \%)$ para la evaluación de la memoria visual, el Trail Making Test - Parte $\mathrm{B}^{(22)}(11,4 \%)$ para evaluar la memoria de trabajo y la secuenciación y el subtest de Clave semántica del Test de Barcelona $^{(23)}(11,4 \%)$ para la evaluación de la fluencia verbal.

A excepción de los dominios de inteligencia, memoria verbal y pensamiento abstracto, en los que sólo se observa un deterioro leve, en los dominios restantes 
Tabla 7. Porcentajes de deterioro cognitivo por dominio

\begin{tabular}{|l|c|c|}
\hline \multicolumn{1}{|c|}{ DOMINIO COGNITIVO } & Deterioro cognitivo leve (\%) & Deterioro cognitivo severo (\%) \\
\hline Inteligencia: & 2,9 & 0 \\
\hline Atención: & & \\
- General. & 5,7 & 28,6 \\
- Selectiva. & 23,5 & 8,8 \\
\hline Memoria: & 5,7 & 11,4 \\
- Memoria de trabajo. & 14,4 & 22,9 \\
- Memoria visual. & 5,7 & 0 \\
- Memoria verbal. & 8,6 & 14,2 \\
\hline Lenguaje: & 5,7 & 5,7 \\
\hline Visuoconstrucción: & 11,4 & 31,4 \\
\hline Velocidad de procesamiento: & & \\
\hline Funciones ejecutivas: & 2,9 & 0 \\
- Pensamiento abstracto. & 5,8 & 11,4 \\
- Planificación. & 2,9 & \\
- Secuenciación. & & \\
\hline
\end{tabular}

Tabla 8. Tabla correlaciones

\begin{tabular}{|c|c|c|c|c|}
\hline & $\begin{array}{c}\text { Depresión } \\
\text { (SDS) }\end{array}$ & $\begin{array}{c}\text { Ansiedad } \\
\text { (STAI) }\end{array}$ & Edad & $\begin{array}{c}\text { Atención selecti- } \\
\text { va (Test de Tolou- } \\
\text { se- Piéron) }\end{array}$ \\
\hline $\begin{array}{c}\text { Velocidad de procesa- } \\
\text { miento (ROCFT) }\end{array}$ & & & $\begin{array}{c}r=-0,373 \\
(p=0,027)\end{array}$ & $\begin{array}{c}r=0,420 \\
(p=0,013)\end{array}$ \\
\hline Fallos Cognitivos (CFQ) & & $\begin{array}{c}r=-0,409 \\
(p=0,015)\end{array}$ & \\
\hline $\begin{array}{c}\text { Atención selectiva (Test } \\
\text { de Tolouse- Piéron) }\end{array}$ & $\begin{array}{c}r=-0,373 \\
(p=0,030)\end{array}$ & & $\begin{array}{c}r=-0,348 \\
(p=0,044)\end{array}$ & \\
\hline
\end{tabular}

se observa la presencia de deterioro cognitivo severo, aún siendo en algunos casos reducido.

A continuación, en la tabla 7 se recogen los porcentajes de deterioro cognitivo leve y severo por dominio cognitivo.

Una vez obtenidos estos resultados se ha hecho un análisis de correlaciones de Pearson entre los distintos tests neuropsicológicos, las variables psicológicas de ansiedad, depresión y percepción de faIlos cognitivos y por último algunas de las variables sociodemográficas (edad y nivel de estudios). Nuestro principal interés en este análisis era estudiar la relación entre el funcionamiento cognitivo objetivo y subjetivo, así como la relación de estas dos variables con la presencia de malestar emocional (ansiedad y depresión).

El análisis de correlaciones muestra la ausencia de relación entre los resultados obtenidos mediante las pruebas neurocognitivas y el cuestionario de fallos cognitivos, de acuerdo con la segunda hipótesis formulada. Son muy pocas las variables que alcanzan una correlación significativa. 
En la tabla 8 se muestra una tabla con las contingencias significativas $(p<0,05)$.

En esta tabla se observa que altos niveles de sintomatología depresiva correlacionan con un rendimiento empobrecido en atención selectiva $(r=-0,373, p=0,030)$, mientras que altos niveles de ansiedad correlacionan con una mayor percepción de fallos cognitivos $(r=-0,409, p=0,015)$.

También podemos observar que las mujeres de mayor edad tienen un rendimiento más pobre en atención selectiva $(r=-0,348, p=0,044)$ y velocidad de procesamiento que las mujeres jóvenes $(r$ $=-0,373 ; p=0,027)$.

\section{Discusión y conclusiones}

Los resultados disponibles en la actualidad revelan que un porcentaje elevado de mujeres presentan un rendimiento similar a la población normal en la primera evaluación. Estos porcentajes oscilan entre un $62 \%$ y un $100 \%$ para cada uno de los dominios cognitivos evaluados, a excepción de la memoria visual, donde sólo un $43 \%$ de la muestra presenta un rendimiento normal.

Estos resultados confirmarían parcialmente la hipótesis formulada, según la cual se esperaba que el rendimiento cognitivo del grupo de pacientes antes de iniciar el tratamiento oncológico sistémico fuera similar a la población normal, siguiendo una distribución normal.

Sin embargo, a pesar de que el rendimiento de un porcentaje elevado de mujeres es normal para la mayoría de dominios cognitivos, también se observa un porcentaje de deterioro cognitivo superior al esperado en la línea base y en una población normal, lo que no permite defender al completo la anterior hipótesis.

En estudios previos que siguen un diseño longitudinal también se ha observado la presencia de deterioro cognitivo en la línea base. Concretamente, Wefel et al. ${ }^{(9)}$ concluyeron que el deterioro cognitivo puede estar presente antes del inicio de los tratamientos, por lo que recomiendan realizar una evaluación previa al inicio del mismo para saber con certeza que cambios a nivel cognitivo pueden atribuirse a los tratamientos y cuáles no. Su resultados indican que el 35\% de la muestra presenta deterioro cognitivo, siendo el aprendizaje verbal $(18 \%)$ y la memoria $(25 \%)$ los dominios más deficitarios.

En cambio, los resultados de nuestro estudio muestran que los dominios con más deterioro son la memoria visual y la atención.

Como ya se mencionaba en la introducción, los estudios de Hurria et al. ${ }^{(12)}$ y de Hermelink et al. ${ }^{(13)}$ también muestran la presencia de deterioro cognitivo en la línea base. Hurria et al. ${ }^{(12)}$ muestran que un $11 \%$ de la muestra presentaba deterioro cognitivo, siendo la memoria visual, la función espacial, la atención y la función psicomotora los dominios más afectados. Hermelink et al. ${ }^{(13)}$ observan que un tercio de las pacientes presentaba deterioro cognitivo en línea base.

En contraposición a nuestra hipótesis, el deterioro cognitivo observado cumple criterios de deterioro severo en determinados dominios cognitivos. Según la hipótesis, en la línea base no se esperaba encontrar deterioro o se esperaba que este fuera de carácter leve, específico de ciertos dominios cognitivos y experimentado por un número muy reducido de mujeres.

En el anterior estudio de Wefel et al. ${ }^{(9)}$ también se observa una relación significativa positiva entre el malestar emocional y el deterioro cognitivo, mientras que en nuestro estudio no se observa relación. Esta falta de correlación entre el malestar emocional y el funcionamiento cognitivo ha sido observada en muchos de los estudios previos ${ }^{(8,28,29)}$ Estos resultados sugieren que la presencia de deterioro cognitivo no se explicaría por la presencia de malestar 
emocional, debiendo de considerar otras variables para su explicación.

A su vez, los resultados muestran que un $73,6 \%$ de mujeres experimenta síntomas depresivos leves o moderados, y un $74 \%$ experimenta niveles de ansiedad dentro de la normalidad. Estos resultados concuerdan con los datos que muestra la literatura ${ }^{(30)}$.

En consonancia con los estudios realizados sobre el tema ${ }^{(6,8,28,31)}$, no se ha encontrado una correlación significativa entre la percepción subjetiva del funcionamiento cognitivo y los tests neuropsicológicos, lo que iría en la línea de la segunda hipótesis formulada. Aunque la persona verbaliza problemas de atención, dificultad para recordar palabras, hacer diversas actividades simultáneamente, etc, su rendimiento en las pruebas neurocognitivas puede ser plenamente normal, no observándose deterioro.

Esta discrepancia entre las medidas objetivas y subjetivas del funcionamiento cognitivo también se ha observado en estudios con pacientes no oncológicos ${ }^{(32,33)}$.

Una posible explicación a estos resultados es que los tests neurocognitivos y los cuestionarios diseñados para evaluar la percepción subjetiva del funcionamiento cognitivo evalúan constructos diferentes. De hecho, el cuestionario de fallos cognitivos correlaciona con otros cuestionarios que evalúan la metamemoria y/o conciencia metacognitiva, es decir, la habilidad general para reflexionar sobre los propios procesos cognitivos, siendo consciente de sus limitaciones ${ }^{(26)}$.

A su vez, también parece que la percepción subjetiva del funcionamiento cognitivo mantiene una fuerte relación con la presencia de malestar emocional ${ }^{(28,34,35)}$ y de una afectividad negativa ${ }^{(31)}$, entendida como la disposición a experimentar sentimientos negativos como culpa, vergüenza, irritabilidad u hostilidad. Estas variables también han mostrado ser independientes del deterioro cognitivo objetivo. Esta relación entre las medidas que evalúan el estado emocional (ansiedad y depresión) y aquellas que evalúan el funcionamiento cognitivo percibido se ha puesto de manifiesto en otros contextos médicos, como por ejemplo con pacientes con $\mathrm{VIH}^{(36)}$.

Otros factores que parecen estar implicados en la percepción subjetiva del funcionamiento cognitivo son el nivel de autoestima y autoeficacia, el pesimismo y ciertos estilos cognitivos como el locus de control. Sabemos que la baja autoestima, así como el pesimismo, conllevan a una tendencia a infravalorar las capacidades propias (físicas y mentales) y la habilidad o recursos para hacer frente a los problemas (autoeficacia) ${ }^{(37,38)}$. En base a ésto es esperable que las personas con baja autoestima, poca confianza en el funcionamiento de su propia mente $y / o$ pesimistas manifiesten más quejas cognitivas que las personas con altos niveles de autoestima, autoeficacia u optimistas.

Del mismo modo, atendiendo al modelo teórico de J. Rotter ${ }^{(39)}$ sobre el constructo de locus de control, sabemos que las personas con un locus de control interno atribuyen sus logros a su conducta o cualidades personales, mientras que las personas con un locus de control externo los atribuyen a la suerte, al azar o acción de otras personas. Lefcourt ${ }^{(40)}$ extrapoló el constructo de locus de control a todo tipo de consecuencias, sean éstas consideradas como logros/refuerzos o no. Estos modelos nos conducen a pensar que aquellas personas con un locus de control interno negativo (sentimiento de falta de habilidades o pérdida de dominio) tenderán a infravalorar su funcionamiento cognitivo, realizando más quejas cognitivas que restantes personas.

Por lo tanto podríamos concluir que la percepción subjetiva sobre el funcionamiento cognitivo es un fenómeno de una gran complejidad y que seria interesante 
poder estudiar con más profundidad en nuevas investigaciones.

En relación a la edad, los resultados de este trabajo indican que ésta es una variable moduladora del rendimiento en las pruebas de atención selectiva y velocidad de procesamiento. Las mujeres de mayor edad obtienen un peor rendimiento en estos dominios. Cimprich et al. ${ }^{(41)}$ realizaron un estudio en el que concluyeron que las pacientes de mayor edad tenían un rendimiento peor en las pruebas de atención antes del tratamiento y que esto conllevaba una mayor vulnerabilidad al deterioro tras la finalización del tratamiento. Hurria et al. ${ }^{(12)}$ y Rodin et al. ${ }^{(42)}$ también estudian la relación entre el funcionamiento cognitivo y la edad en mujeres con cáncer de mama, obteniéndose resultados contradictorios.

Los resultados obtenidos en esta investigación hacen replantearse si el diseño del estudio y las pruebas neuropsicológicas utilizadas para la evaluación de los distintos dominios cognitivos son las más adecuadas. A pesar de ello, la presencia de deterioro cognitivo o un rendimiento disminuido en la línea base defiende la necesidad de realizar una evaluación pre-tratamiento. La ausencia de esta línea base puede conducir a una infravaloración o sobrevaloración del deterioro cognitivo inducido por la quimioterapia.

En relación a este tema, hay que considerar que la presencia de un rendimiento disminuido o anormal frente a puntuaciones basadas en valores normativos, en función de la edad y de los años de escolarización en la primera evaluación no implica necesariamente que el rendimiento sea un déficit o pérdida respecto a una situación previa. El concepto de deterioro o déficit cognitivo requiere implícitamente de una evaluación longitudinal. En una única evaluación transversal es normal que algunas personas tengan un rendimiento inferior al del resto de las personas, y este no tiene porque ser considerado anormal, al igual que personas que rinden por encima de 1,5 DE. Estas personas siempre tendrán puntuaciones inferiores al resto sin que ello signifique que exista una pérdida. Este aspecto queda muy bien representado en la curva de la normalidad o campana de Gauss, que ejemplifica el rendimiento de una población que sigue una distribución normal.

El alto porcentaje de deterioro cognitivo podría ser debido a diversos problemas metodológicos inherentes al diseño, como son:

- La falta de idoneidad de las pruebas neuropsicológicas empleadas para la población objeto de estudio. Esto puede conducir a una infraestimación o sobrestimación del deterioro cognitivo. Concretamente, la Figura Compleja ${ }^{(20)}$ es una prueba que puede estar generando un importante número de falsos positivos en memoria visual, debido a la complejidad que esta prueba supone para muchas de las mujeres evaluadas. En esta prueba primero se solicita al paciente que copie un dibujo geométrico y después se le pide que lo reproduzca de memoria. Un porcentaje reducido de mujeres, pero superior al esperado, tienen dificultades para copiar el dibujo, lo que inevitablemente repercute en el recuerdo que posteriormente tiene. Si la praxis es mala, es esperable que la memoria también lo sea. Por este motivo, convendría retirar esta prueba de la batería y sustituirla por otras alternativas que se encuentren menos sesgadas por el nivel educativo y que permitan evaluar de forma independiente la memoria visual y la capacidad visuoconstructiva. Entre estas alternativas tenemos la utilización de los Cubos de Kohn de la Escala de Inteligencia para adultos - $3^{\text {a }}$ versión ${ }^{(17)}$ u el Subtest de Copia de una Figura de la Batería para la Evaluación del Estado Neuropsicológico $\left(\right.$ RBANS) ${ }^{(43)}$ para la evaluación de la praxis constructiva y el Subtest de Dibujos de la 
Escala de Memoria - $3^{a}$ edición ${ }^{(17)}$ y Subtest de Recuerdo Demorado de la Figura del RBANS $^{(43)}$ para la memoria visual.

— Un alto porcentaje de mujeres (83\%) ha sido evaluado tras el primer ciclo de quimioterapia, a pesar de que el diseño metodológico contemplaba realizar la primera evaluación antes de iniciar el tratamiento. En muchas ocasiones el tiempo transcurrido entre la visita del oncólogo y el inicio de la quimioterapia era inferior a 48 horas, lo que dificultaba su evaluación previa. Estas mujeres fueron a pesar de todo evaluadas, considerando que el perjuicio de la quimioterapia sobre el funcionamiento cognitivo, no es inmediato, sino que tiene lugar tras unos ciclos de quimioterapia ${ }^{(7)}$ pero con ello se está incumpliendo uno de los criterios de exclusión establecidos en el estudio. Aún cuando se considera que el deterioro cognitivo asociado a la quimioterapia sobre el funcionamiento cognitivo no es inmediato, no se dispone de datos claros al respecto. Además, el hecho de estar bajo el tratamiento con quimioterapia hace que la persona no se encuentre físicamente bien, lo que también puede repercutir en los resultados, independientemente del poder neurotóxico que la quimioterapia haya podido ejercer. De cara a proseguir con este estudio convendría buscar la forma de contactar y evaluar a las mujeres antes de su primer ciclo de quimioterapia, siendo de este modo como tendremos datos reales de su funcionamiento premórbido. Es esperable que si evaluamos a las mujeres antes de iniciar el tratamiento los porcentajes de deterioro serán más reducidos.

- La fatiga mental producida por la duración de la evaluación. Aún cuando las mujeres manifiesten no estar cansadas, seria conveniente: 1.- Detener la evaluación durante un período de 15 minutos transcurridos aproximadamente los primeros 90 minutos de evaluación, que coincide con la finalización del K-BIT ${ }^{(15)}$; 2.- Seleccionar un conjunto de pruebas neurocognitivas que permitan evaluar los diversos dominios cognitivos en un tiempo inferior a 90 minutos.

- El déficit visual experimentado por algunas pacientes. Este déficit ha influido en algunas de las pruebas administradas, fundamentalmente en el test de atención ${ }^{(21)}$. Algunas mujeres se olvidan de traer las gafas y realizan las pruebas con cierto sobreesfuerzo, lo que conduce a un peor rendimiento ya que muchas de estas pruebas tienen un tiempo límite de ejecución. Los datos de estas mujeres han sido incluidos en el análisis lo que ha podido inflar el número de mujeres con deterioro. De cara a proseguir con el estudio será importante recordar a las mujeres que acudan con sus gafas y en el caso de que no las traigan no considerar sus resultados en las pruebas que se puedan ver sesgadas por el déficit visual.

- Los criterios establecidos para determinar la presencia de deterioro cognitivo. A pesar de considerar, tras una revisión profunda del tema, que los criterios empleados en nuestro análisis son los más idóneos, en posteriores análisis de los datos se puede considerar el empleo de diversos criterios, observando como difieren las proporciones de deterioro obtenidas. Esta variabilidad en los porcentajes de deterioro en función de los criterios considerados para establecer su presencia en el análisis se ha puesto de manifiesto en diversos estudios ${ }^{(44,45,46)}$

En el estudio de Desai et al. ${ }^{(44)}$, en el que se evaluaba el funcionamiento cognitivo previo al inicio de la quimioterapia en mujeres con cáncer de mama, se observó que el deterioro cognitivo variaba entre un $15,2 \%$ y un $26,1 \%$ en función de los criterios empleados.

Del mismo modo, en el estudio de Shilling(45), también se concluyó que la proporción de deterioro cognitivo en una muestra de mujeres con cáncer de mama 
transcurrido un mes desde la finalización de la quimioterapia variaba de entre un $12 \%$ a un $68,5 \%$ en función de las siete definiciones y criterios empleados.

Un estudio algo más reciente ${ }^{(46)}$ también muestra que las proporciones de deterioro son función de los criterios empleados (cuatro criterios más o menos estrictos), así como de los diferentes grupos de referencia considerados a la hora de analizar los datos. Tomando como referencia los datos normativos publicados se observa que la proporción de deterioro en mujeres con cáncer de mama antes de iniciar el tratamiento oncológico variaba de entre $1 \%$ a $36,6 \%$ según el criterio empleado. Mientras que en relación a un grupo control formado por mujeres sanas, la proporción de deterioro oscilaba entre $13,7 \%$ y $45,4 \%$.

Todos estos factores pueden haber ejercido un rol importante en los resultados obtenidos con esta muestra por lo que antes de proseguir con el estudio deberán considerarse y buscar un modo de controlarlos.

De este estudio, se pueden extraer importantes implicaciones clínicas y de investigación.

Como implicaciones clínicas se debe destacar la importancia de prestar atención y recoger las quejas cognitivas expresadas por los pacientes, y el desarrollo de programas de rehabilitación cognitiva destinados a los supervivientes de cáncer, que muestran dificultades a nivel cognitivo, tanto a través de medidas objetivas como subjetivas.

En cuanto a las implicaciones de investigación, es importante incluir una evaluación neuropsicológica previa a la iniciación de los tratamientos, sirviendo como línea base del funcionamiento cognitivo, con la que comparar las puntuaciones obtenidas en posteriores evaluaciones. Como hemos podido observar, algunas mujeres muestran deterioro antes de iniciado el tratamiento. De no haber realizado está primera evaluación, en posteriores evaluaciones se hubiera producido una sobrestimación del deterioro cognitivo, atribuyéndolo al efecto adverso de los tratamientos.

También es importante que se alcance un acuerdo respecto a los criterios empleados para establecer la presencia de deterioro cognitivo, así como de los tests utilizados para su evaluación. En cuanto a los criterios seria bueno que todos los investigadores analizásemos los resultados siguiendo unos mismos criterios, de forma que pueden compararse las proporciones obtenidas en diferentes estudios de forma adecuada. Respecto a los tests, éstos deberían ser sensibles para detectar un deterioro cognitivo leve y tener la suficiente validez ecológica para identificar los problemas que las pacientes experimentan en su vida cotidiana. También seria importante que los tests utilizados estuvieran adaptados y estandarizados en todos los idiomas, de forma que los distintos investigadores del tema, independientemente de su país, pudiesen utilizar unos mismos instrumentos de evaluación, lo que favorecería la comparación de resultados.

A su vez, convendría que los tests establecidos para explorar la presencia de deterioro cognitivo asociado a los tratamientos oncológicos en pacientes con cáncer constituyeran una batería más breve, con mayor aplicabilidad clínica.

Por último, sería importante sensibilizar a los profesionales sanitarios que trabajan con pacientes oncológicos sobre la presencia de este fenómeno e informarles de los resultados que se obtienen de las investigaciones, así como darles herramientas de evaluación en el contexto de la entrevista médica que junto con su juicio clínico les permita detectar su presencia. 


\section{REFERENCIAS BIBLIOGRÁFICAS}

1. Berglund G, Bolund C, Fornander, T, Rutqvist LE, Sjödén PO. Late effects of adjuvant chemotherapy and postoperative radiotherapy on quality of life among breast cancer patients. Eur J Cancer 1991; 27: 107581. Doi:10.1016/0277-5379(91)90295-O

2. Wieneke MH, Dienst ER. Neuropsychological assessment of cognitive functioning following chemotherapy for breast cancer. Psychooncology 1995; 4 (1): 61-6. Doi:10.1002/pon.2960040108

3. van Dam FSAM, Schagen SB, Muller MJ, Boogerd W, Wal, E vdl, Fortuyn MED, et al. Impairment of cognitive function in women receiving adjuvant treatment for hight-risk breast cancer: High-dose versus standard dose chemotherapy. J Natl Cancer Inst. 1998; 90: 210-8. Doi:10.1093/ jnci/90.3.210

4. Schagen SB, van Dam FSAM, Muller MJ, Boogerd W, Lindeboom J, Bruning PF. Cognitive deficits after postoperative adjuvant chemotherapy for breast carcinoma. Cancer 1999; 85: 640-50. doi:10.1002/(SICl)1097$0142(19990201) 85: 3<640:$ : AID CNCR14>3.0.CO;2-G

5. Brezden CB, Phillips KA, Abdolell M, Bunston T, Tannock IF. Cognitive function in breast cancer patients receiving adjuvant chemotherapy. J Clinical Oncol 2000; 18: 2695-701.

6. Ahles TA, Saykin AJ, Furstenberg CT, Cole B, Mott LA, Skalla K et al. Neuropsychologic impact of standard-dose systematic chemotherapy in long-term survivors of breast cancer and lymphoma. J Clin Oncol 2002; 20 (2): 485-493. Doi:10.1200/ JCO.20.2.485

7. Tchen N., Juffs HG., Downie FP., Yi QL, $\mathrm{Hu} \mathrm{H}$, Chemerynsky I, et al. Cognitive function, fatigue and menopausal symptoms in women receiving adjuvant chemotherapy for breast cancer. J Clin Oncol 2003; 21 (22): 4175-83. Doi:10.1200/ JCO.2003.01.119
8. Castellon SA, Ganz PA, Bower JE, Petersen L, Abraham L, Greendale GA, et al. Neurocognitive performance in breast cancer survivors exposed to adjuvant chemotherapy and tamoxifen. J Clin Exp Neuropsychol 2004; 26 (7): 955-69. Doi:10.1080/13803390490510905

9. Wefel JS, Lenzi R., Theriault RL, Davis $\mathrm{RN}$, Meyers CA. The cognitive sequelae of standard-dose adjuvant chemotherapy in women with breast carcinoma: Results of a prospective, randomized, longitudinal trial. Cancer 2004; 100 (11): 2292-9. Doi:10.1002/cncr.20272

10. Shilling V, Jenkins V, Morris R., Deutsch D, Bloomfield D. The effects of adyuvant chemotherapy on cognition in women with breast cancer - preliminary results of an observational longitudinal study. Breast 2005; 14(2): 142-50. Doi:10.1016/j. breast.2004.10.004

11. Bender CM, Sereika SM, Berga SL, Vogel VG, Brufsky AM, Paraska KK, et al. Cognitive impairment associated with adyuvant therapy in breast cancer. Psychooncology 2006; 15 (5): 422-430. Doi:10.1002/ pon.964

12. Hurria, A., Rosen, C., Hudis, C., Zuckerman, E. Panageas KS, Lachs MS, Witmer M, et al. Cognitive function of older patients receiving adjuvant chemotherapy for breast cancer: A pilot prospective longitudinal study. J Am Geriatr Soc 2006; 54: 925-31. Doi:10.1111/j.1532-5415.2006.00732.x

13. Hermelink K, Untch M, Lux M, Kreienberg $R$, Kreienberg R, Beck $T$, et al. Cognitive function during neoadjuvant chemotherapy for breast cancer. Results of a prospective, multicenter, longitudinal study. Cancer 2007; 109(9): 1905-913. Doi 10.1002/ cncr.22610

14. Benedet MJ, Alejandre MA. Test de Aprendizaje Verbal España-Complutense (TAVEC). Madrid: TEA, 1998.

15. Cordero A, Calonge I. Test Breve de Inteligencia de Kaufman K-BIT. Versión Española. Madrid: TEA Ediciones, 2000. 
16. Delis DC, Kramer JH, Kaplan E, Ober BA. The California Verbal Learning Test. San Antonio, TX: Psychological Corporation California, 1987

17. Wechsler D. Escala de Inteligencia para adultos, $3^{a}$ versión. España: TEA Ediciones, 2001.

18. Zung WW. A self-rating depression scale. Arch Gen Psychiatry 1965: 12: 63-70.

19. Alonso J, Regidor E, Barrio G, Prieto L, Rodriguez C, de la Fuente L. Cuestionario de Salud SF-36. Barcelona: Institut Municipal d’Investigació Mèdica, 203.

20. Meyers JE, Meyers KL. Rey Complex Figure Test. Psychological Assessment Resources, Inc, 1995

21. Toulouse E, Piéron H. T-P Prueba perceptiva y de atención. Madrid: Ediciones TEA, 1998.

22. Reitan RM, Wolfson D. The HalsteadReitan Neuropsychological Test Battery: Theory and clinical interpretation (2nd ed). Tucson, AZ: Neuropsychology Press, 1993

23. Peña-Casanova J. Test de Barcelona- Revisado. Programa Integrado de Exploración Neuropsicológica. Barcelona: ElsevierMasson, 2005.

24. Spielberger CD, Gorsuch RL, Lushene RE. Cuestionario de Ansiedad Estado - Rasgo. Madrid: TEA Ediciones, 1986.

25. Cella DF, Tulsky DS, Gray G, Sarafian B, Linn, Bonomi $A$, et al. The Functional Assessment of cancer Therapy (FACT) Scale: Development and validation of the general version. J Clin Oncol 1993; 11(3):570-9.

26. Broadbent DE, Cooper PF, FitzGerald P, Parkes KR. The Cognitive Failures Questionnaire (CFQ) and its correlates. $\mathrm{Br}$ J Clin Psychol, 1982, 21 (Pt1): 1-16. Doi:10.1111/j.2044-8260.1982.tb01421.x

27. Molinuevo Guix, JL. Deterioro cognitivo leve. Antecedentes históricos y concepto. España: Editorial Glosa, 2007. p.: 9-23.

28. Jenkins V, Shilling V, Fallowfield L, Howell A, Hutton S. Does hormone therapy for the treatment of breast cancer have a detrimental effect on memory and cognition? A pi- lot study. Psychooncology, 2004; 13: 61-6. Doi:10.1002/pon.709

29. Shilling V, Jenkins V. Self-reported cognitive problems in women receiving adjuvant therapy for breast cancer. Eur J Oncol Nurs 2007; 11: 6-15. Doi:10.1016/j. ejon.2006.02.005

30. Poppelreuter M, Weis J, Kulz AK, Trucha O, Lange KW, Bartsch $\mathrm{HH}$. Cognitive dysfunction and subjective complaints of cancer patients. A cross-sectional study in a cancer rehabilitation centre. Eur J Cancer, 2004; 40(1):43-9. Doi:10.1016/j. ejca.2003.08.001

31. Hemerlink $K$, Küchenhoff $H$, Untch $M$, Bauerfeind I, Lux MP, Bühner $M$ et al. Two different sides of "chemobrain": determinants and nondeterminants of self-perceived cognitive dysfunction in a prospective, randomized, multicenter study. Psychooncology, 2010; 19: 1321-8. Doi: 10.1002/ pon.1695

32. Radziwillowicz W, Radziwillowicz P. Subjective and objective assessment of memory functions in endogenous depression. Arch Psychiatry Psychotherapy, 2000; 2: 33-41.

33. Millikin $\mathrm{CP}$, Rourke $\mathrm{SB}$, Halman $\mathrm{MH}$, Power C. Fatigue in HIV/AIDS is associated with depression and subjective neurocognitive complaints but not neuropsychological functioning. J Clinical Exp Neuropsychol, 2003; 25 (2): 201-15. Doi:10.1076/ jcen.25.2.201.13644

34. Servaes P, Verhagen CA, Bleijenberg G. Relations between fatigue, neuropsychological functioning and physical activity alter treatment for breast carcinoma. Daily self-report and objective behavior. Cancer 2002; 95 (9): 2017-26. Doi:10.1002/ cncr.10891

35. Pullens M, De Vries, J, Roukema J. Subjective cognitive dysfunction in breast cancer patients: a systematic review. Psychooncology 2010; 19: 1127-138. Doi:10.1002/pon.1673

36. Saykin AJ, Janssen R, Sprehn G Kaplan J E, Spira T J, O'Connor Bl. Longitudinal eva- 
luation of neuropsychological function in homosexual men with HIV-1 infection: 18 month follow-up. J Neuropsychiatry Clin Neurosci, 1991; 3: 286 -98.

37. Branden N. Los seis pilares de la autoestima. Barcelona: Paidós, 1995.

38. André C, Lelord F. La Autoestima. Gustarse a sí mismo para mejor vivir con los demás. Barcelona: Editorial Kairós, 1999.

39. Rotter JB. Generalized expectancies for internal versus external control of reinforcement. Psychological Monograph 1966, 80,1-28. Doi:10.1037/h0092976

40. Lefcourt HM. Personality and locus of control. In: Garber J, Seligman MEP, editor. Human helplessness: Theory and applications. New York: Academic Press, 1980. p. 24559.

41. Cimprich B., So H., Ronis DL, Trask C. Pre-treatament factors related to cognitive functioning in women newly diagnosed with breast cancer. Psychooncology 2005; 14: 70-8. Doi:10.1002/pon.821

42. Rodin MB, Wallace JA, Lacy M, Kuball K, Pykkonen B, Fleming G. Does adjuvant chemotherapy (CT) exacerbate cognitive impairment in elderly breast cancer (BC) patients? Results of a prospective, longitudinal study. J Clin Oncol, 2006. ASCO Annual Meeting Proceedings, Vol. 24, No 18S (June 20 Supplement): 10530.

43. Randolph C. Repeatable Battery for the Assessment of Neuropsychological Status (RBANS), 1998.

44. Desai J, Wagner LI, Beaumont JL, Sweet J, et al. Cognitive functioning among breast cancer patients prior chemotherapy. Abstract. Psychooncology, 2005: 14: S1-S104 (P2-6).

45. Shilling V, Jenkins V, Trapala IS. The (mis) classification of chemo-fog? Methodological inconsistencies in the investigation of cognitive impairment after chemotherapy. Breast Cancer Res Treat 2005; 95 (2): 1259. Doi:10.1007/s10549-005-9055-1

46. Schilder CM, Seynaeve C, Linn SC, Boogerd W, Gundy CM, Beex LV, et al. The impact of different definitions and reference groups on the prevalence of cognitive impairment: a study in postmenopausal breast cancer patients before the start of adjuvant systemic therapy. Psychooncology 2010; 19: 415-22. Doi:10.1002/ pon. 1595 